\title{
НАПРАВЛЕНИЯ И ПОТЕНЦИАЛ АВТОМАТИЗАЦИИ ПРОЦЕССОВ В ОМНИКАНАЛЬНОМ КОНТАКТ-ЦЕНТРЕ ПРОВАЙДЕРА ТЕЛЕКОМ-УСЛУГ
}

\section{FIELDS AND PROSPECTS OF PROCESS AUTOMATION IN A TELECOM PROVIDER'S OMNI-CHANNEL CONTACT CENTRE}

M. Kapustkin

Summary. Following extensive digital trends in the mass service provision, telecom industry contact centers (CC) take robust advantages of both omni-channel customer care and routine job automation experience. This paper spotlights fields of the routine business process automation in a telecom provider's traditional model-featured omnichannel CC. For each of the fields introduced, underlaying technologies of software solution framework are described, then a predictive estimation of the achievable business process automation scale is provided. Crucial constraints of complete automation approach are considered as based on takeaways of earlier researches, exploration of available software solutions too. Because flexible professional techniques are critical to enhance the customer satisfaction with CC services, and certain risks of non-constructive innovation management still exist, the importance of gradual advances in business task automation is primarily suggested.

Keywords: automaton; routine process; contact center; customer service; omni-channel interaction; telecom industry; business innovations; autonomous system; artificial intelligence; artificial neural network; virtual assistant, etc.

\author{
Капусткин Максим Владимирович \\ Санкт-Петербургский Государственный \\ Политехнический Университет Петра Великого \\ kapustkin.mv@edu.spbstu.ru
}

Аннотация. Поддерживая технологические тенденции в сфере массового обслуживания, контакт-центры (КЦ) провайдеров телеком-услуг реализуют переход к омниканальному сервису и внедряют средства автоматизации рутинных задач. В предлагаемой статье рассмотрены направления автоматизации бизнес-процессов в рамках стандартной операционной модели омниканального КЦ предприятия телеком-отрасли. Для каждого из направлений предложен обзор технологических аспектов ІТ-решений и приведена концептуальная оценка возможностей автоматизации ассоциированных с ним процессов. На основе результатов исследований и анализа характеристик современного программного обеспечения для центров обслуживания установлены факторы, ограничивающие потенциал всеобъемлющей автоматизации. С учётом гибкой реализации отдельных функций, рисков избыточного и нескоординированного внедрения инноваций подчёркивается необходимость планомерного, сопровождающегося поэтапным увеличением масштаба задач перехода к модели автономного КЦ.

Ключевые слова: автоматизация; рутинный процесс; контакт-центр; клиентское обслуживание; омниканальное взаимодействие; телеком-индустрия; бизнес-инновации; автономная система; искусственный интеллект; искусственная нейронная сеть; виртуальный ассистент и др.

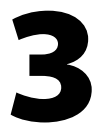

а десятилетия утверждения и развития методик улучшения потребительских практик (Customer Experience, CX) услуги контакт-центров (КЦ) стали неотъемлемой частью организации публичного сервиса как на основе институциональных, так и деловых стандартов взаимодействия с инициаторами обращений (заявителями, действующими и потенциальными клиентами). Массовая востребованность КЦ обусловлена удобством и оперативностью дистанционного формата связи, получившего особую значимость (а в отдельных сегментах рынка услуг оказавшегося безальтернативным) с началом пандемии COVID-19 [11]. Глобальный прогноз [19] фиксирует ожидания устойчивого роста оборота и стоимости услуг КЦ (на уровне 5-6\% в годовом выражении) вплоть до завершения указанного в исследовании периода (2020-2027 гг. включительно).
Рутинный характер большинства запросов и высокий спрос на дистанционные сервисы, предоставляемые компаниями массового рынка, в т.ч. провайдерами телеком-услуг, определяют актуальность инициатив по внедрению технологий автоматизации задач, до недавнего времени выполнявшихся персоналом профильных отделов: подготовка электронной отчётности, эскалация жалоб и протоколов инцидентов, сбор статистики о причинах обращений и т.д. Программное обеспечение, успешно заменяющее операторов в обработке типовых запросов (информирование о балансе, статусе заявок, заказ продукции и др.), даёт стимул к масштабной трансформации бизнес-процессов, сопровождающейся освоением новых, высокотехнологичных подходов к выполнению функций КЦ [12]. Согласно результатам исследования [10], мировой рынок решений для автоматизации КЦ ждёт 
двузначный ежегодный прирост оборота (около 18\%) с близким к 4-кратному увеличением общей стоимости контрактов за рассмотренный период (2020-2025 гг. включительно). В свою очередь, итоги отраслевого мониторинга [55] [56], проведённого в странах СНГ, отчётливо указывают на заинтересованность ведущих менеджеров в применении технологий автоматизации: до 2022 года выполнять с их помощью не менее 50\% операций, связанных с CX, планирует каждый пятый участник опроса, в то время как важность интеграции тех же решений для улучшения ключевых показателей бизнеса подтвердили свыше $80 \%$ респондентов.

Исходя из приведённых фактов, технологическую трансформацию следует рассматривать как долгосрочный вызов, определяющий актуальность изучения текущих и перспективных изменений в модели КЦ с традиционным доминированием человеческих ресурсов. В частности, в данном исследовании предпринята попытка выделения и анализа направлений внедрения, а также потенциала развития IT-решений для автоматизации процессов на базе омниканального (англ. omnichannel) КЦ телеком-провайдера, предоставляющего унифицированную платформу сервиса с обработкой обращений, полученных по телефону, через системы контроля заявок клиентов (CRM) и приложения экспресс-коммуникации в сети Интернет (социальные медиа, мессенджеры, онлайн-форумы, системы видеосвязи). К настоящему времени поддержка омниканальности во взаимодействии с аудиторией рассматривается как стандартная практика, в значительной мере влияющая на оценку уровня и качества оказания услуг КЦ [58]. С учётом внимания к данному аспекту среди исследователей и представителей делового сообщества интерес к специфике автоматизации с учётом омниканальности, обозначенный в работе, следует назвать способствующим более полному и конструктивному обзору актуальной тенденции.

Предлагаемая работа включает введение, представление основных релевантных источников, основную часть, заключение и список литературы. Материал основной материал состоит из 3 разделов. В разделе 1 устанавливаются мотивы перехода к модели инновационного омниканального КЦ провайдера телеком-услуг, приводятся базовые характеристики распространённых подходов к её внедрению, выделяются критерии для оценки эффективности проектов по развитию платформы нового типа. В разделе 2 определены направления автоматизации процессов, соответствующие модели специализации трудовых ресурсов типового центра обслуживания. В разделе 3 отмечены особенности решений, соответствующих направлениям автоматизации задач КЦ в рамках телеком-отрасли, зафиксированы важнейшие недостатки и ближайшие цели для улучше- ний существующих программных средств. Выводы к основному содержимому обзора изложены в заключении.

\section{Основные исследования}

Трансформация операционной модели КЦ в условиях бума IT-разработок отражает генеральную тенденцию, характерную как для сферы услуг, так и для всех отраслей современной экономики. В этой связи среди значимых публикаций последнего времени превалируют работы, обобщающие опыт мониторинга и подготовки прогнозов автоматизации в различных сегментах массового обслуживания. В глобальном исследовании [21], наряду с макроконъюнктурой цифровых преобразований в государственном и корпоративном секторах, анализируются масштаб и динамика внедрения автоматических программных комплексов в системы предприятий-операторов услуг. Обоснование предпосылок и прогнозы ускоренной автоматизации непроизводственных процессов, прежде всего в сфере работы с клиентами, представлены в материалах аналитических агентств Forrester [37] и Gartner [49].

Более детальный анализ факторов и направлений трансформаций в модели КЦ доступен в статьях Jose и др. [27], Kirkpatrick [29], Saberi и др. [42] ежегодных общемировых [6] [10] [19] и региональных [55] [56] обзорах конъюнктуры и тенденций отрасли.

Анализ технологических факторов и перспектив улучшения метрик обслуживания в компаниях телеком-индустрии, в т.ч. при взаимодействии с клиентами на базе КЦ, проведён Imbug и др. [23], Johansson и др. [26]. В статье Rendón и др. [40] введена модель измерения качества услуг, предоставляемых клиентам телеком-провайдеров, с учётом эффективности поддержки, количества и доступности дистанционных каналов связи.

Преимущества и технологические аспекты предоставления омниканального сервиса на платформе КЦ раскрыты в исследовании Золотухиной и Голубевой [58]. Участниками проекта [17] предложен прототип программного фреймворка, унифицирующего приём и обработку обращений через оффлайн- и онлайн-каналы взаимодействия с аудиторией. В работе Suciu и др. [46] изложен опыт организации омниканального центра обслуживания с автоматизацией задач при ведении диалога с клиентом в социальной сети и через мессенджер.

Значительная часть публикаций, отвечающих актуальной теме и включённых в список источников, содержит описания индивидуальных и групповых проектов по внедрению программных решений для автоматизации различных функций операторов и аналитиков КЦ. 


\section{口 Операционно-исполнительские}

- Рост производительности в рамках бизнес-процессов

- Оптимизация задач операторов

- Повышение ценности (оценки) операций среди потребителей

- Повышение ценности результатов бизнес-процессов

\section{口 Функционально-технологические}

- Масштабируемость решений

- Быстрая функциональная адаптация решений

\section{Э Экономические}

- Сокращение издержек на предоставление сервиса

- Реакция на опыт конкурентов

\section{口 Гуманитарные}

- Содействие психологическому комфорту операторов

Рис. 1. Стимулы внедрения (развития) технологических инноваций на базе КЦ.

Преимущественно на их основе выделены общеотраслевые и ориентированные на телеком-провайдеров разработки, подвергнутые обзору в основной части (раздел «Особенности и потенциал программных решений»). Экспериментальные системы на основе технологий автоматического распознавания (Automatic Speech Recognition, ASR) и синтеза (Text-to-Speech Technology, TTS) речи предложены в статьях Khandelwal и др. [28], Tarasiev и др. [48], Vasilateanu и др. [50]. Практический потенциал систем искусственного интеллекта (ИИ) на основе искусственных нейронных сетей (ИНС), находящий применение в т.ч. в автоматизации задач КЦ, продемонстрирован в работах Suciu и др. [46], Völkle и др. [51], Wang и др. [52]. Варианты реализации программных комплексов для сбора и анализа больших данных (Big Data) на базе КЦ представлены в работах Chanpanit и др. [5], Stepanov и др. [45], Неустроева [60]. Возможности алгоритмов машинного обучения в адаптации нейронных сетей, позволяющих вести анализ и контроль потока обращений в КЦ, проработаны в исследованиях Albrecht и др. [2], Hernandez и др. [22], Mateo [33].

\section{1. Автоматизация в работе КЦ: перехо к п^атформе инновачий}

\section{1. Стимулы}

Автоматизацию традиционного КЦ, включая подразделения компаний телеком-индустрии, можно рас- сматривать как один из вариантов характерного для цифровой эпохи бизнес-кейса, предполагающего как однозначную формулировку целей, так и всесторонний анализ факторов, влияющих на выбор подхода и средств для достижения требуемого эффекта. Вид и масштаб оказываемых услуг, а также культура взаимодействия с их получателями обусловливают специфические глубину и сложность интеграции решений в организованные на базе отделов и центров обслуживания IT-системы. Несмотря на отсутствие универсальных триггеров для применения средств автоматизации, аналитический отчёт [55], подготовленный в Национальной ассоциации контактных центров (НАКЦ), позволяет зафиксировать ключевые предпосылки ухода от традиционной модели сервиса. Результаты мониторинга, сформированные путём сравнения данных глобального и регионального (в пределах стран СНГ и Украины) опросов представителей отрасли, наглядно свидетельствуют о значимости 4 групп стимулирующих факторов (рис. 1).

Основываясь на диаграмме, приводимой в отчёте, можно констатировать, что наиболее актуальными мотивами запуска и развития программ автоматизации КЦ оказываются операционно-исполнительские (рост производительности, оптимизация труда операторов), экономические (минимизация операционных издержек) и гуманитарные (обеспечение психологического комфорта сотрудников). При этом качествен- 
Таблица 1. Подходы к автоматизации процессов КЦ.

\begin{tabular}{|c|c|c|c|}
\hline Наименование подхода & Предпосылки внедрения & Сложности внедрения & Примеры \\
\hline $\begin{array}{l}\text { Автоматизация локальных } \\
\text { задач }\end{array}$ & $\begin{array}{ll}\text { - } & \text { выполнение рутинных } \\
\text { задач на отдельных этапах } \\
\text { выбранного процесса } \\
\text { потребность в сохранении } \\
\text { базовой схемы и логики } \\
\text { операций выбранного } \\
\text { процесса }\end{array}$ & $\begin{array}{ll}\text { - } & \text { анализ локальных } \\
& \text { зависимостей } \\
\text { и интерфейсов передачи } \\
\text { данных } \\
\text { - } & \text { достижение } \\
\text { совместимости } \\
\text { с технологическим стеком } \\
\text { в рамках одного процесса }\end{array}$ & \begin{tabular}{|l} 
- \\
маршрутизация входящих \\
вариантов интерактивного \\
голосового меню (англ. \\
Interactive Voice Response, \\
IVR) \\
показ информации \\
из профиля клиента \\
в системе обработки \\
звонков по результатам \\
идентификации его номера \\
\end{tabular} \\
\hline $\begin{array}{l}\text { Автоматизация потоков } \\
\text { задач }\end{array}$ & \begin{tabular}{|l} 
поддержка процессов / \\
подпроцессов, сводимых \\
(на уровне этапов или \\
в целом) к сериям задач \\
рутинного типа \\
необходимость \\
оптимизации объёма / \\
сложности операций \\
в ведении сотрудников \\
при условии обеспечения \\
их дальнейшего участия \\
в конкретном процессе
\end{tabular} & $\begin{array}{ll}\text { - } & \text { организация } \\
\text { непрерывных (бесшовных) } \\
\text { переходов между } \\
\text { задачами в структуре } \\
\text { потока } \\
\text { настройка приёма } \\
\text { и передачи данных } \\
\text { между подразделениями } \\
\text { (функциями), } \\
\text { задействованными } \\
\text { в обработке одного } \\
\text { потока }\end{array}$ & $\begin{array}{l}\text { - } \quad \text { автоматическая оценка } \\
\text { качества обработки } \\
\text { звонков / запросов } \\
\text { с предварительным } \\
\text { распознаванием и анализом } \\
\text { речи / текста в сеансе } \\
\text { общения } \\
\text { формирование прогноза } \\
\text { объёма и интенсивности } \\
\text { звонков / запросов в ходе } \\
\text { автономного мониторинга } \\
\text { характеристик потока } \\
\text { обращений (общее } \\
\text { количество, средняя } \\
\text { длительность обслуживания, } \\
\text { количество на 1 сотрудника } \\
\text { и др.) }\end{array}$ \\
\hline $\begin{array}{l}\text { Автоматизация каналов } \\
\text { коммуникаций }\end{array}$ & 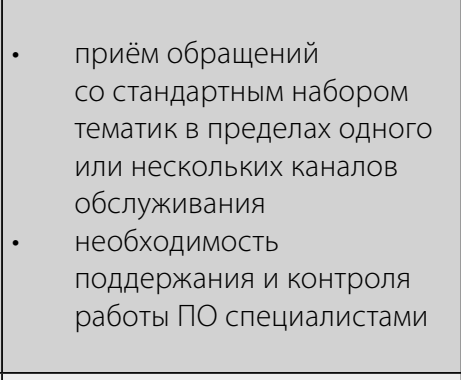 & $\begin{array}{ll}\text { - } & \text { адаптация к специфике } \\
\text { языка и сценариев } \\
\text { коммуникации в рамках } \\
\text { предметной области } \\
\text { достижение скорости } \\
\text { и конструктивности } \\
\text { реакции на уровне, } \\
\text { аналогичном или } \\
\text { максимально близком } \\
\text { человеческому общению }\end{array}$ & $\begin{array}{l}\text { интеграция голосовых } \\
\text { помощников и чат-ботов } \\
\text { для обработки звонков } \\
\text { и обращений через онлайн- } \\
\text { каналы связи }\end{array}$ \\
\hline Роботизация исполнителей & \begin{tabular}{|l} 
- \\
руачитинельная доля \\
автоматизации задач среди \\
реализуемых в рамках \\
выбранного подразделения \\
или функции \\
опыт успешной интеграции \\
решений для автоматизации \\
потоков задач и/или \\
каналов коммуникаций
\end{tabular} & $\begin{array}{l}\text { - } \quad \text { настройка операций } \\
\text { с программным } \\
\text { окружением, } \\
\text { сформированным } \\
\text { для учёта обращений, } \\
\text { создания отчётов, } \\
\text { аналитики и т.д. } \\
\text { адаптация алгоритма } \\
\text { к нестандартным } \\
\text { ситуациям (конфликтам, } \\
\text { прецедентным практикам } \\
\text { потребителей и т.д.) }\end{array}$ & 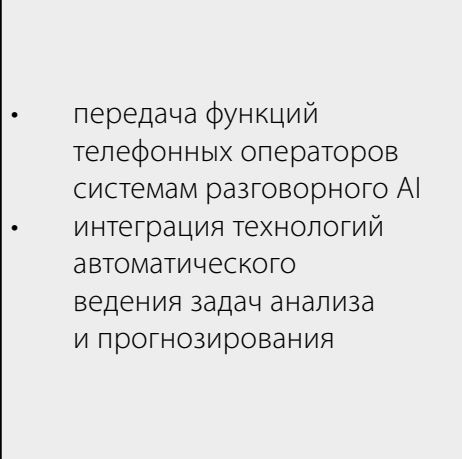 \\
\hline
\end{tabular}


Таблица 1. Подходы к автоматизации процессов КЦ (продолжение).

\begin{tabular}{|c|c|c|c|}
\hline Наименование подхода & Предпосылки внедрения & Сложности внедрения & Примеры \\
\hline $\begin{array}{l}\text { Автоматизация } \\
\text { операционной платформы }\end{array}$ & $\begin{array}{l}\text { • } \\
\text { высокий потенциал } \\
\text { автоматизации всех } \\
\text { основных бизнес- } \\
\text { процессов в действующей } \\
\text { модели КЦ } \\
\text { опыт успешного внедрения } \\
\text { средств автоматизации } \\
\text { исполнительских функций / } \\
\text { каналов коммуникаций / } \\
\text { потоков задач }\end{array}$ & \begin{tabular}{|l} 
- \\
определение и анализ \\
технологических \\
подсистем (уровня \\
подразделений, групп) \\
согласно бизнес-логике \\
для платформы нового \\
типа \\
оценка полноты \\
и качества перехода \\
с учётом числа, \\
структурной специфики \\
и глубины взаимодействия \\
подсистем в платформе \\
нового типа \\
\end{tabular} & $\begin{array}{l}\text { - } \quad \text { адаптация комплексных } \\
\text { решений на базе облачных } \\
\text { платформ RingCentral [9], } \\
\text { Five9 [15], Genesys [16] } \\
\text { и других IT-вендоров }\end{array}$ \\
\hline $\begin{array}{l}\text { Интеграция автономного } \\
\text { КЦ-решения }\end{array}$ & $\begin{array}{l}\text { • } \\
\text { низкая производительность } \\
\text { потенцимальный } \\
\text { существующей } \\
\text { технологической } \\
\text { платформы КЦ } \\
\text { • необходимость } \\
\text { оперативного } \\
\text { развёртывания услуг КЦ без } \\
\text { привлечения штатных или } \\
\text { внешних (через договор } \\
\text { аутсорсинга) исполнителей }\end{array}$ & $\begin{array}{l}\text { - } \\
\text { отсутствие полного } \\
\text { программного } \\
\text { обеспечения для } \\
\text { реализации функций } \\
\text { центра обслуживания; } \\
\text { риск несовместимости } \\
\text { с ранее интегрированным } \\
\text { ПО сторонних вендоров } \\
\text { (системы управления } \\
\text { предприятием, заявками } \\
\text { клиентов и др.) }\end{array}$ & $\begin{array}{l}\text { в } \\
\text { сиедренем, предлагаемых } \\
\text { Replicant [41], Neuro. } \\
\text { net [59] и другими } \\
\text { специализированными } \\
\text { вендорами }\end{array}$ \\
\hline
\end{tabular}

ные аспекты IT-решений, в частности обеспечение масштабируемости, отличает меньшая релевантность, что объясняется преобладающей экспериментальностью проектов по внедрению передовых технологий игроками отрасли, продолжающими оценивать практические эффекты автоматизации с учётом первичных требований (производительность, ресурсоэффективность, удобство использования, рентабельность). Следует ожидать, что по мере комплексного анализа и углубления базового опыта функциональные преимущества, отличающие предложения IT-вендоров, будут приобретать всё большую значимость как стимул к полной трансформации платформы и модели обслуживания.

\section{2. Подходы}

Опыт автоматизации внутренних процессов в действующих КЦ описан в многочисленных прикладных исследованиях [22] [27] [34]. Используя предложенный в них анализ проблемных факторов и вариантов технологических улучшений, а также сведения о текущих решениях IT-вендоров для провайдеров омниканального сервиса [7] [16] [41], возможно выделить несколько подходов, практикуемых в рамках различных стратегий освоения инноваций:
- автоматизация локальных задач ориентирована на полностью автономное проведение отдельных операций или этапов в рамках процессов, входящих в зону ответственности конкретного подразделения КЦ;

- автоматизация потоков задач (workflow) предусматривает обеспечение программного контроля над цепочкой операций, имеющих локальный (в рамках одного подразделения) или кросс-функциональный (с обменом информацией между подразделениями) характер;

- автоматизация каналов взаимодействия связана с внедрением программ, заменяющих сотрудников КЦ в рамках контактов с аудиторией по одному или нескольким каналам коммуникации;

- роботизация исполнителей проявляется в отказе от человеческих ресурсов, задействованных для выполнения стандартного набора операций (в составе подразделения или профильной группы), в пользу роботизированных процессов (англ. Robotics Process Automation, RPA);

- автоматизация операционной платформы основана на масштабном, продолжительном внедрении инноваций, гарантирующих автономную реализацию всех процессов в модели центра обслуживания; 
- интеграция автономного КЦ-решения достигается приобретением и настройкой доступа к удалённой (облачной) платформе, предоставляющей технологии разговорного ИИ (conversational Al) и набор сопутствующих сервисов (распознавание речи, аналитика обращений, управление маршрутизацией и др.) при обеспечении основной функциональности высококачественного имитирования практик обработки звонков/запросов операторами физического КЦ.

Предпосылки и потенциальные сложности внедрения, а также примеры использования каждого из подходов представлены в таблице 1.

Исходя из общей логики, положенной в основу подобного видения, целесообразно зафиксировать связь представленных подходов с масштабами проектов автоматизации. Сложность организации и ведения бизнес-процессов в омниканальном КЦ, входящем в структуру провайдера, диктует внедрение комплексных и подчас дорогостоящих решений, что, в свою очередь, обусловливает конструктивность поступательного перехода с уровня локальных операций до автоматизации функций, структурных подразделений и в перспективе - всей системы задач центра обслуживания. В то же время, в зависимости от практических результатов и стратегии развития, в рамках отдельных подсистем (например, процессов уровня подразделений) могут применяться комбинированные подходы (в частности, для отдела обслуживания стоит считать ожидаемой автоматизацию одного или нескольких каналов сервиса при одновременном внедрении роботизированного оператора).

\section{3. Критерии эффективности}

Общие признаки эффективности шагов по внедрению и поддержке инноваций в КЦ следует рассматривать аналогичными учитываемым в большинстве проектов по интеграции автономных IT-решений для нужд учреждений и предприятий. Факторы, заложенные в основу данных признаков, связаны с соответствием практических результатов автоматизации следующим критериям:

- достижение целевого уровня автоматизации (полная или частичная)

- выполнение требований (функциональных / нефункциональных) к итоговой системе или компоненту в рамках выбранного объектом автоматизации процесса (серии процессов)

- соблюдение ограничений на доступность ресурсов (финансовых, временных, человеческих и т.д.)
Регулярность работы, характерная для КЦ компаний телеком-индустрии, устанавливает особые требования к срокам реализации и контроля качества настройки решений для автоматизации ключевых процессов обслуживания и смежных функций. В проектах, охватывающих сервисы с особым режимом доступа (на основании запросов правоохранительных органов, экстренных, надзорных служб и т.д.), принципиальным характером обладают производительность и надёжность обработки данных при взаимодействии по любому из поддерживаемых каналов связи.

Успешность интеграции систем AI и RPA в поддержку омниканального сервиса (вплоть до автоматизации всего штата операторов) определяется точностью установления, корректностью и скоростью обработки информации (текстовой, речевой), т.е. эффективность проектов в данном сегменте инноваций зависит, прежде всего, от степени адаптации существующих технологий к специфике взаимодействия с клиентами провайдера. Этим обстоятельством обусловлен дополнительный набор метрик контроля качества (точность / задержка распознавания, средний интервал реагирования и др.), приводимых в текущих и итоговом отчётах проектных команд.

Результаты глобального опроса, проведённого Cisco [6], демонстрируют усиление внимания к аспектам информационной безопасности (ИБ), в частности защиты персональных данных пользователей услуг КЦ. С учётом данного факта следует ожидать, что набирающая обороты автоматизация КЦ в телеком-секторе будет сопровождаться всё более комплексными требованиями по минимизации рисков утечки данных и критических для всей операционной платформы уязвимостей ПО.

\section{2. Направления \\ проектов автоматизации}

В настоящее время на рынке автоматических технологий для КЦ телеком-провайдеров, как и в предложениях для других отраслей, наблюдается доминирование решений, направленных на минимизацию потребности в человеческих ресурсах, привлекаемых к выполнению задач внутри конкретных подразделений (отделов) компании. Ассортимент профильных вендоров (Five9, RingCentral, Voximplant и др.) отражает распределение исполнительских функций, соответствующее типовой организационной модели современных центров дистанционного обслуживания. Основываясь на данной модели и понимании обстоятельств, обусловливающих рутинное выполнение различных функций, можно выделить ряд направлений с характерными составами объектов и технологий разработки средств автоматизации процессов КЦ: 
Таблица 2. Охватываемые процессы и состав технологий в направлениях автоматизации КЦ.

\begin{tabular}{|c|c|c|}
\hline Направление автоматизации & Процессы & Основные используемые технологии \\
\hline Клиентское обслуживание & \begin{tabular}{|l} 
приём и обработка обращений \\
клиентов по предоставленным \\
каналам связи (телефония / CRM / \\
етаil / онлайн-сервисы) \\
передача обращений в смежные \\
отделы (в случае прецедента, \\
эскалации, особого статуса клиента \\
и т.д.) \\
подготовка служебных отчётов \\
по факту оказания услуг
\end{tabular} & $\begin{array}{ll}\text { - } & \mathrm{Al} \\
\text { - } & \mathrm{ML} \\
\text {. } & \mathrm{DL} \\
\text {. } & \mathrm{ASR} \\
\text { TTS }\end{array}$ \\
\hline Контроль качества & $\begin{array}{ll}\text { - } & \text { проверка соответствия качества } \\
\text { обслуживания клиентов внутренним } \\
\text { регламентам } \\
\text { подготовка служебных отчётов } \\
\text { по результатам профильных операций }\end{array}$ & $\begin{array}{ll}\text { - } & \mathrm{ML} \\
\text { - } & \mathrm{ASR} \\
\text { - } & \mathrm{BDP}\end{array}$ \\
\hline Анализ и планирование & 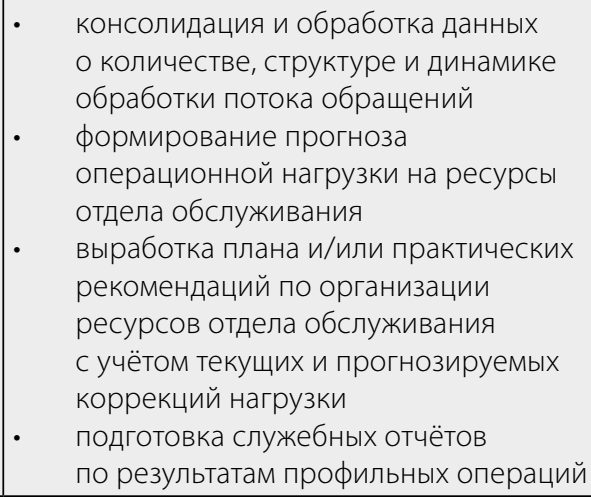 & $\begin{array}{ll}\text { - } & \mathrm{Al} \\
\text { - } & \mathrm{ML} \\
\text { - } & \mathrm{BDP}\end{array}$ \\
\hline Администрирование IT-ресурсов & 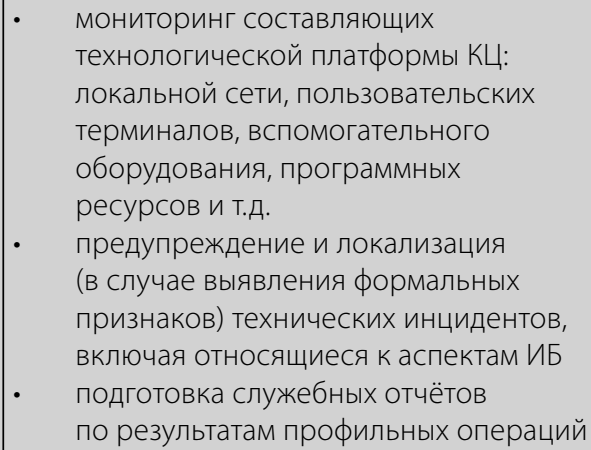 & $\begin{array}{ll}\text { - } & \mathrm{Al} \\
\text { - } & \mathrm{ML}\end{array}$ \\
\hline
\end{tabular}

* Условные обозначения:

Al - Artificial Intelligence (искусственный интеллект)

$\mathrm{ML}$ - Machine Learning (машинное обучение)

DL - Deep Learning (глубокое обучение)

ASR - Automatic Speech Recognition (автоматическое распознавание речи)

TTS - Text-to-Speech (синтез речи)

BDP - Big Data Processing (обработка больших данных)

\footnotetext{
- автоматизация клиентского обслуживания

- автоматизация контроля качества

- автоматизация анализа и планирования

- автоматизация IT-администрирования
}

Структура объектов автоматизации (процессов) и технологий, достигших наибольшей актуальности и масштабов применения в каждом из направлений, представлена в таблице 2 .

Проводя общий анализ, необходимо подчеркнуть, что автоматизация в любом из направлений лишена зависимости от реализации аналогичных проектов в том или ином подразделении предприятия. Внедре- 
ние специализированных решений для автономного контроля качества или обработки данных, обладающих ценностью для поддержки функций планирования, может достигаться при сохранении традиционной организации процессов в отделе обслуживания (т.е. в условиях приоритетного использования труда операторов). В текущем представлении данный тезис согласуется с подходом, предполагающим переход основного набора задач конкретного подразделения (профильной группы) под контроль роботизированных программных систем. Тем не менее, утверждая основным методом вывода направлений анализ конъюнктуры автоматизации в исследуемой области, следует констатировать, что сопоставленные им процессы отражают актуальность поступательного, а не одномоментного ввода инноваций. Специфика телеком-услуг, определяемая их технической сложностью и зачастую неоднозначным восприятием характеристик со стороны клиентов, диктует необходимость более гибких, комбинированных подходов (начиная с уровня отдельных задач) к автоматизации функций обслуживания. В то же время многообразие факторов, учитываемых при анализе и прогнозировании нагрузки на операторов (в частности, рост абонентской базы вследствие сделок с крупными предприятиями), создаёт риски неэффективной роботизации планирования, по крайней мере на раннем этапе применения подобной системы. Обосновывающие ту же идею примеры ситуаций возможно привести, сосредоточиваясь на аспектах управления качеством и IТ-ресурсами КЦ.

Оценка программных решений, рассмотренных далее, в значительной мере аргументирует целесообразность поэтапного внедрения и увеличения масштаба инноваций в каждом из вышеуказанных направлений.

\section{3. Особенности и потеншиал программных решений}

\section{1. Клиентское обслуживание}

Следуя потребности в обеспечении и развитии омниканального взаимодействия, разработчики решений для автоматизации задач операторов предлагают средства, учитывающие специфику обслуживания при ведении как голосовой, так и текстовой коммуникации с клиентами [12]. В то время как качественные распознавание и синтез речи, проводимые в режиме телефонного диалога, основаны на применении целого комплекса технологий, среди которых выделяются ИИ и нейронные сети глубокого обучения (Deep Neural Network, DNN), результативная и признаваемая удовлетворительной реакция на запросы, поступающие через публичные чаты и мессенджеры, всё чаще достигается путём внедрения программ-ассистентов (ботов) с типовыми алгоритмами и наборами функций [30] [51]. Сопутствующие сервисы, поддерживающие автоматический мониторинг и учёт статистики обращений для подготовки индивидуальных и групповых отчётов, предоставляются рядом вендоров в рамках пакетных предложений, в частности вызывающих широкий интерес CCaaS-решений (Contact Center as a Service) на базе облачных технологий обработки и хранения данных [24].

Решающими факторами роботизации телефонного сервиса следует назвать точность распознавания и скорость синтеза речи с применением специализированных программных комплексов. На текущий момент в сегменте ASR/TTS-решений представлено большое число мультиязычных систем с различной степенью соответствия обоим критериям, прежде всего обеспечению минимально возможной доли ошибок в наборе распознанных слов (Word Error Rate, WER) [57]. Возможности отдельных технологий (Google Speech API, Yandex SpeechKit), поддающихся быстрой интеграции и демонстрирующих сравнительно высокие результаты обработки разговорной речи, получают развитие в академических проектах, позволяющих оценить потенциал автоматического распознавания на основе прикладных моделей с воссозданием структуры и логики бизнес-процессов. Функциональность системы поиска и вывода подсказок, представленной в статье Vasilateanu и др. [50], достигается на всём протяжении потокового анализа диалога оператора с клиентом телеком-провайдера. В проекте по разработке экспериментальной системы «Twin» [48], организованной путём интеграции обеих вышеуказанных технологий в единый модуль распознавания, определены релевантными и отражены в итоговом решении особенности, характерные для обмена информацией в реальном времени, В т.ч. и при звонках в КЦ (различная длительность фраз, наличие шума в канале связи, чередующиеся упоминания имён, данных о времени и др.). Указанные работы, а также другие исследования последних лет (преимущественно в области адаптации инструментов ASR/TTS к обработке аудиопотоков на региональных языках) могут рассматриваться как свидетельство придания научной важности аспектам качества распознавания и синтеза речи, их роли в разработке и развитии средств автоматизации бизнес-процессов.

Наиболее перспективные реализации ASR/TTS, coставляющие основу абсолютного большинства решений современных вендоров, представляют примеры синергии искусственных нейронных сетей (ИНС) и технологий машинного обучения (к таким примерам относятся и ранее упомянутые продукты Google и Яндекс). Объективные преимущества ИНС в сравнении с сугубо алгоритмическим подходом проявляются не только в способности адаптироваться к сложным случа- 
ям, до сих пор предполагавших обязательное участие человека, но и в возможности передачи его функций роботизированным (виртуальным) операторам, гарантирующим параллельное обслуживание всех каналов доступа к услугам КЦ [20]. В подобных обстоятельствах особое внимание исследователей и вендоров уделено выбору наиболее эффективных моделей ИНС, а также их интеграции в системы разговорного ИИ, поддерживающие целевой контекст обмена данными в режиме реального времени. Результаты, приводимые рядом авторов [18] [30] [52], подтверждают практическую ценность и доступность улучшений соответствующих систем для максимально реалистичного ведения диалога в широком спектре каналов связи.

Прогресс, достигнутый в проектах по созданию прототипов и промышленных моделей роботов для КЦ, не следует считать исчерпывающим. До настоящего времени углублённого изучения и всестороннего учёта при разработке решений требуют несколько факторов. Во-первых, критическими недостатками всех технологий ASR остаются низкая точность распознавания и необходимость повторения фраз, произнесённых с явным акцентом [28] или заметными речевыми дефектами (ситуация может неконтролируемо усугубляться фоновым шумом [25]). Во-вторых, как убедительно показано в исследовании Szymański и др. [47], общепризнанные бенчмарки, используемые в оценке корректности результатов ASR, совершенно не отражают сложность задач, возникающих при обработке информации во время телефонного разговора. В-третьих, серьёзные сомнения вызывает адаптивность виртуальных операторов к уровню эмоциональности фраз, с учётом которого определяется модель конструктивного ведения диалога (для текущих бенчмарков распознавания эмоций актуальны те же противоречия, что и для используемых в измерении точности перевода речи в текст).

Учитывая значительную сложность и трудоёмкость исследований каждого из указанных аспектов, можно утверждать, что полная автоматизация задач, до настоящего времени выполняемых операторами КЦ, влечёт риски снижения СХ-результатов обработки специфических и требующих подчёркнуто гибкой реакции обращений (прежде всего в контексте услуг, оказываемых по телефону). Тем не менее, качественная интеграция, как и повышение инновационной составляющей решений, ориентированных на автоматическую обработку звонков / запросов рутинного характера (справочная поддержка, проверка ограничений на доступ к сервисам связи и др.), способствуют развитию тенденции к предельному уменьшению роли человеческих ресурсов в регулярном обслуживании. Наиболее показательные достижения в современной бизнес-практике, В частности применение собственного робота-опера- тора в КЦ банка Тинькофф [54], характеризуются высокой деловой эффективностью и обнаруживают существенный потенциал для функционального углубления автоматизации.

\section{2. Контроль качества}

За последние несколько лет в системе организации контроля качества (Quality Assurance, QA) услуг КЦ, предлагаемых клиентам телеком-предприятий, были достигнуты существенные успехи, позволяющие рассматривать данное направление как одно из наиболее перспективных с точки зрения практической полноты и эффективности автоматизации. Активно развиваемые инноваторами Computer Science технологии ASR, машинного обучения и Big Data послужили основой многочисленных фреймворков, исключающих или сводящих к минимуму потребность в ручном управлении и коррекции настроек ПО. Возможности и результаты интеграции подобных систем продемонстрированы в целом ряде проектов [32] [38] [60]. Генеральная логика, характерная для фреймворка автоматизации контроля качества, предполагает компьютерную обработку текстов с результатами распознавания речи в разговоре оператора с абонентом (в работе Mariappan и др. [32] анализу подвергаются сообщения участников чате). Сопоставляя текущий состав фраз ключевым словам и выражениям, указывающим на признаки (не)соответствия регламенту ведения диалога с клиентом, система оценивает степень удовлетворения критериям качества, формируя детальный отчёт. Для эффективной агрегации и хранения больших данных в программных комплексах применяются технологии распределённых вычислений и Big Data (Hadoop, MapReduce, Hive и др.) [13] [45] [60].

Аналогичные или концептуально близкие реализации, предлагаемые в виде отдельных разработок и в рамках пакетных решений различных вендоров, имеют очевидный потенциал массового применения в КЦ, включая подразделения компаний телеком-индустрии. Вместе с тем, следует констатировать, что действующими факторами, ограничивающими полноту и выгоды автоматизации в направлении QA, выступают как риски ошибочного или частичного распознавания фраз, так и техническая сложность выявления контекста, влияющего на ход общения и, вследствие этого, специфику представления информации оператором. С учётом обоих факторов программные системы оценки качества, как и решения для роботизированного обслуживания, сохранят актуальность для минимизации числа рутинных процессов и приводимых к ним цепочек операций (сбор материалов для контроля, структурный анализ разговора / переписки и т.д.). При этом полную автоматизацию процедур QA допустимо полагать ориентиром и потенциальным результатом 
проектных усилий на пересечении технологий ИИ, распознавания речи и машинного обучения.

\section{3. Анализ и планирование}

Как и в направлении, связанном с контролем качества, автоматизация задач анализа и планирования ресурсов КЦ охватывает абсолютное большинство рутинных процессов, отличаясь высоким уровнем проработки и активным улучшением технологических фреймворков. Ключевыми элементами функциональности, реализуемой в данном сегменте решений, следует назвать перманентные сбор и обработку данных о конъюнктуре потока обращений (объём, интенсивность и распределение по каналам коммуникаций) для автоматического прогнозирования нагрузки на ресурсы отдела обслуживания (операторов и компоненты локальной сети). Ввиду значительного размера и комплексного состава входных данных, консолидирующих статистику по обращениям различных типов, в качестве универсальных составляющих технического дизайна систем применяются технологии Big Data [5] [39] [45]. В то же время стандартной практикой моделирования и разработки становится реализация нейросетевого подхода с усовершенствованным распознаванием признаков и оценкой изменений операционной нагрузки в режиме реального времени [2] [24].

Отсутствие субъективности и нечёткости в исходных данных, использование программируемых алгоритмов исследования и интерпретации результатов позволяют рассматривать подобные решения как способствующие автоматизации оперативного анализа и подготовки краткосрочных планов (рекомендаций) по поддержанию производительности основных процессов КЦ. Более того, в условиях массовой доступности и стабильной востребованности телеком-услуг, существенно ограничивающих влияние конъюнктурных изменений на отрасль и бизнес-процессы компаний [23], интеграцию автоматических систем целесообразно учитывать среди значимых факторов оптимизации более масштабного (в оценке временного интервала) мониторинга и прогнозирования активности клиентской аудитории. Тем не менее, данная перспектива не лишает актуальности опыт и навыки профессионального аналитика, проявляющиеся в грамотном определении и фиксации как стратегических, так и постоянных рисков, связанных с нерегулярными (в частности, при возникновении чрезвычайных ситуаций) всплесками запросов на услуги КЦ.

\section{4. Администрирование IT-ресурсов}

Проекты по автоматизации контроля IT-ресурсов, сосредоточенных на базе КЦ, выделяются в одно из направлений корпоративных стратегий инноваций, реализуемых большинством компаний телеком-индустрии. К текущему моменту, исходя из общей логики построения и развития технологических систем для бизнес-целей, ключевым фактором изменений, достигаемых в масштабе КЦ, следует назвать автоматизацию управления корпоративной сетью, обеспечивающей передачу данных между всеми структурными подразделениями предприятия. К наиболее значимым характеристикам работы сети, предполагающим рутинный контроль со стороны администратора и, вследствие этого, усиливающим интерес к возможностям автоматического мониторинга и предотвращения инцидентов, относятся:

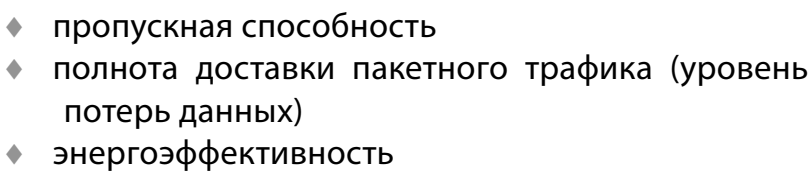

В масштабном исследовании [35], проведённом среди представителей фирм телеком-отрасли, дан обзор передовых практик организации и развития автономно управляемых локальных сетей. Наряду с системами алгоритмического сбора и анализа статистики операций для автоматической оптимизации трафика, авторами отмечены примеры эффективного внедрения решений на основе ИНС (главным образом для выявления и контроля рисков, вызванных задержками передачи и потерями данных). Оценки улучшений, приводимые в обзоре, особенно показательны с учётом ежедневных нагрузок на ресурсы локальных сетей крупных телеком-провайдеров (Vodafone, China mobile и др.), выступающих пионерами в разработках нейросетевого ИИ для администрирования собственных IT-платформ. Подобные эффекты допустимо рассматривать как способствующие росту скорости и качества коммуникаций во всех интегрированных в сеть подразделениях компаний, включая КЦ.

Результаты вышеупомянутого исследования, отражающие преобладание позитивных прогнозов операционных и экономических последствий автоматизации сетевого контроля в телеком-компаниях (в выгодах от её достижения убеждены более трёх четвертей респондентов), позволяют ожидать расширения функциональности профилактики инцидентов при одновременном увеличении масштаба автономно управляемых IT-систем. В зависимости от деловой стратегии ключевым фактором, способным поддержать подобные и более комплексные инновации, может выступать разработка оригинальных программных продуктов, организованная в соответствии со спецификой бизнес-процессов и технологического обеспечения конкретного предприятия. В то же время для обеспечения всеобъемлющей автоматизации в контексте задач системного 
(сетевого) администрирования крайне важна углублённая проработка как сценариев автономного реагирования на технические сбои, так и путей минимизации угроз информационной безопасности. Можно утверждать, что последний аспект наиболее критичен для операций с персональными данными клиентов и, как следствие, определяет особые требования к защищённости систем IT-контроля в пределах КЦ.

\section{Зак^ючение}

Внедрение технологий, минимизирующих долю ручных операций и, тем самым, потребность в человеческих ресурсах, кардинально трансформирует работу омниканальных КЦ, предлагающих услуги клиентам телеком-компаний [1]. Некогда рискованная стратегия автоматизации, поддержанная небольшим числом игроков отрасли, стала безальтернативной для всех предприятий, заинтересованных в высокой производительности и эффективности дистанционного сервиса. Обобщая варианты адаптации КЦ к современным и будущим вызовам, подходы, рассмотренные в данной работе, отражают различные масштабы и сценарии автоматизации процессов внутри центров и отделов обслуживания - от промежуточных операций до интеграции полнофункциональных систем на базе ИНС с возможностью автономного обучения. В то же время, обеспечив полный ассортимент соответствующих инноваций, вендоры специализированного ПО получают стимул к созданию продуктов, учитывающих операционные особенности IT-платформ и приоритеты конкретных бизнес-пользователей. В подобных условиях автоматизация рутинных задач перешла из области стратегического планирования в разряд конъюнктурных потребностей, предполагающих поиск эксклюзивных решений с минимальными рисками технически сложной интеграции.
Вместе с тем, несмотря на солидные характеристики и функциональность современных систем, возможности автоматизации процессов КЦ, связанной с их внедрением, объективно далеки от универсальных. Специфичность (вплоть до применения индивидуальных подходов) реагирования на прецедентные и чрезвычайные обращения клиентов в практике регулярного обслуживания заведомо ограничивает потенциал гибкого поведения роботов, вызывая риски коммерческих и репутационных издержек полной автоматизации услуг операторов. Наиболее предсказуемый характер задач, установленных в других направлениях, не детерминирует превосходящую эффективность программных систем, способных выполнять функции отдельных исполнителей и целых подразделений, но даёт импульс интенсивной оптимизации работы последних (при оценке качества обслуживания, предсказании клиентской активности, координации нагрузки на локальную сеть и т.д.). Текущие недостатки, прослеживаемые в критических для коммуникаций системах распознавания речи, определяют дополнительный аргумент в пользу тщательно проработанного, планомерного перехода от традиционной модели КЦ к информационно-технологической платформе нового типа, нивелирующей влияние рутинных операций на организацию рабочего времени, что особенно актуально для конструктивного применения навыков высококвалифицированных специалистов.

Компетентные выбор и интеграция решений для автоматизации неотделимы от понимания элементов и логики подвергаемых ей процессов. В этой связи перспективным предметом исследований видится подробная характеристика наиболее типовых локальных задач, с уровня которых целесообразно планировать и вести автоматизацию столь комплексных схем операций, как реализуемые в КЦ телеком-компаний.

\section{ЛИТЕРАТУРА}

1. 6 Types of Call Center Automations You Should Have // getvoip.com: In-depth VolP news and analytics platform URL: https://getvoip.com/blog/2019/01/03/ call-center-automations/ (дата обращения: 28.01.2021).

2. Albrecht T., Rausch T.M., Derra N.D. Call me maybe: Methods and practical implementation of artificial intelligence in call center arrivals' forecasting //Journal of Business Research. - 2021.-T. 123.-C. 267-278.

3. Bojanić M., Delić V., Karpov A. Call redistribution for a call center based on speech emotion recognition //Applied Sciences. — 2020.— T. 10.— № . 13.— C. 4653.

4. Call Center Automation Depends on Collaborative AI // customerthink.com: customer experience solutions and strategies URL: https://customerthink.com/ call-center-automation-depends-on-collaborative-ai/ (дата обращения: 28.01.2021).

5. Chanpanit T.,Udomsakdigool A. Big Data Framework for Incoming Calls Forecasting in a Call Center //2020 International Conference on Electrical, Communication, and Computer Engineering (ICECCE). — IEEE, 2020. - C. 1-4.

6. Cisco Contact Center Global Survey 2020 // www.cisco.com: Cisco official website URL: https://www.cisco.com/c/dam/en/us/products/collateral/contactcenter/cc-global-survey-ebook.pdf (дата обращения: 28.01.2021).

7. Cisco solutions to advance contact center automated experience // cisco.com: Cisco official website URL: https://www.cisco.com/c/en/us/products/contactcenter/index.html (дата обращения: 28.01.2021). 
8. Cohen Y., Reis J., Amorim M. Empirical Analysis of Call Center Load \& Service Level for Shift Planning //International Conference on Exploring Services Science. Springer, Cham, 2020.- C. 79-91.

9. Contact Center Platform Overview — RingCentral Software Products // ringcentral.co.uk: RingCentral official website URL: https://www.ringcentral.co.uk/ contact-centre/overview.html\#ring-uk (дата обращения: 28.01.2021).

10. Contact Center Transformation Market - Growth, Trends, Forecasts (2020-2025) // reportlinker.com: search engine for statistical counts, insights and forecasts URL: https://www.reportlinker.com/p05986888/Contact-Center-Transformation-Market-Growth-Trends-Forecasts.html?utm_source=PRN (дата обращения: 28.01.2021).

11. Covid-19 crisis has speeded up contact centre digital transformation // computerweekly.com: news headlines and analysis in digital industries URL: https:// www.computerweekly.com/feature/Covid-19-crisis-has-speeded-up-contact-centre-digital-transformation (дата обращения: 28.01.2021).

12. Creating The Uncommon Customer Experience // tcs.com: IT innovations for business purposes URL: https://www.tcs.com/perspectives/articles/reimaginingcontact-center-digital-technologies (дата обращения: 28.01.2021).

13. Dai J.J. et al. Bigdl: A distributed deep learning framework for big data //Proceedings of the ACM Symposium on Cloud Computing. - 2019. - C. 50-60.

14. Ding L. et al. "Forced Automation" by COVID-19? Early Trends from Current Population Survey Data.— Federal Reserve Bank of Philadelphia, 2020.— № . 88713.

15. Five9 Software Solution Website // five9.com: Five9 official website URL: https://www.five9.com/ (дата обращения: 28.01.2021).

16. Full-featured software solutions for contact center automation // genesys.com: Genesys official website URL: https://www.genesys.com/solutions/customerservice/contact-center (дата обращения: 28.01.2021).

17. Gamess E., Mora F., Oliveros D., Urribarri D. Integrating a Unified Communications System with Social Networks // Revista Venezolana de Computación. 2018. Vol.5, No.1. C. 20-28.

18. Gilbert M. et al. Intelligent virtual agents for contact center automation //IEEE Signal Processing Magazine.— 2005.—T. 22.— № . 5.—C. 32-41.

19. Global Call Centers Industry // reportlinker.com: search engine for statistical counts, insights and forecasts URL: https://www.reportlinker.com/p087286/ World-Call-Centers-Markets.html?utm_source=GNW (дата обращения: 28.01.2021).

20. Han K.J. et al. Deep Learning-Based Telephony Speech Recognition in the Wild //INTERSPEECH. — 2017. — C. 1323-1327.

21. Hawksworth J., Berriman R., Goel S. Will robots really steal our jobs? An international analysis of the potential long term impact of automation // PricewaterhouseCoopers, http://pwc.co.uk/economics, access. - 2018. - T. 13.

22. Hernandez L., Guzman H., Ospino J., Freyle J., Pranolo A. Design and implementation of a Marking Strategy to Increase the Contactability in the Call Centers, Based on Machine Learning // International Journal on Advanced Science, Engineering and Information Technology. 2019. Vol.9, No.1. C. 1-7.

23. Imbug N. et al. The influence of customer experience on customer loyalty in telecommunication industry //International Journal of Academic Research in Business and Social Sciences. - 2018. - T. 8. - № . 3. - C. 103-116.

24. Intelligent automation in the telco contact center // inform.tmforum.org: TM Forum website - Telecom enterprise and e-content provider alliance URL: https://inform.tmforum.org/research-reports/intelligent-automation-in-the-telco-contact-center/ (дата обращения: 28.01.2021).

25. Ivanov A.V., Fazluktinov P.S., Kolesnev V.A. Applying intelligent systems of speech recognition for optimizing the algorithm of noise reduction in audio records // Journal of Physics: Conference Series. 2020. Conf. Ser. 1441 012040. C. 1-10.

26. Johansson T., Kidron T. Improving Customer Experience in Telecommunications Company. — 2017.

27. Jose B., Kumar S.D.M., Cuzzocrea A. Enhancing contact center performance using cloud computing: a case study on telecom contact centers //Proceedings of the Second International Conference on Internet of things, Data and Cloud Computing. - 2017. - C. 1-8.

28. Khandelwal K. et al. Black-box Adaptation of ASR for Accented Speech //arXiv preprint arXiv:2006.13519. — 2020.

29. Kirkpatrick K. Al in contact centers //Communications of the ACM. - 2017. - T. 60. - № . 8. - C. 18-19.

30. Kurachi Y., Narukawa S., Hara H. Al chatbot to realize sophistication of customer contact points //Fujitsu Scientific \& Technical Journal. — 2018. — T. 54.— № . 3.- C. 2-8.

31. Manoharan R., Ganesan R., Kumar K. A study of business and operational performances of call centers using speech technology // International Journal on Advanced Science, Engineering and Information Technology. 2015. № Vol.10, №.1. C. 6601-6610.

32. Mariappan R. et al. QART: A Tool for Quality Assurance in Real-Time in Contact Centers //Proceedings of the 25th ACM International on Conference on Information and Knowledge Management. - 2016.-C. 2493-2496.

33. Mateo R.M.A.A Knowledge Extraction Framework for Call Center Analytics //Online World Conference on Soft Computing in Industrial Applications. — Springer, Cham, 2014.-C. 129-141.

34. Mononen M. et al. Robotic Process Automation and its effect on contact center agents' work and resourcing. - 2020.

35. Network automation using machine learning and AI // inform.tmforum.org: TM Forum website — Telecom enterprise and e-provider alliance URL: https:// inform.tmforum.org/research-reports/network-automation-using-machine-learning-and-ai/ (дата обращения: 28.01.2021).

36. Özlan B., Haznedaroğlu A., Arslan L.M. Automatic Fraud Detection In Call Center Conversations //2019 27th Signal Processing and Communications Applications Conference (SIU). - IEEE, 2019.-C. 1-4.

37. Predictions 2021: Customer Service // forrester.com: survey and analytics services URL: https://www.forrester.com/fn/16sPtw302SdUjlxUYTXCAs (дата 06paщения: 28.01.2021).

38. Raju K.V.K., Manaswini V.N.S. Analyzing Call Data Through Live Calls Using Sphinx Tool //International Journal of Engineering \& Technology. — 2018. — T. 7.— №. 3.31.-C. 93-97. 
39. Rashid 0. et al. Intelligent decision making and planning for call center //2019 International Conference on Computer and Information Sciences (ICCIS).—IEEE, 2019.-C. 1-6.

40. Rendón C.M.C. et al. Proposed Model for Measuring Customer Satisfaction with Telecommunications Services //Mediterranean Journal of Social Sciences. 2017. - T. 8. - № . 2. - C. 15-15.

41. Replicant Voice Contact Center Features// replicant.ai: Replicant official website URL: https://www.replicant.ai/products/replicant-voісе/ (дата обращения: 28.01.2021).

42. Saberi M., Hussain 0., Chang E. Past, present and future of contact centers: a literature review // Business Process Management Journal. 2017. № Vol.23, No.3. C. 574-597.

43. Schneider S., Kokshagina 0. Digital transformation: What we have learned (thus far) and what is next //Creativity and Innovation Management. - 2021. T. 1. - № . 1.-C. 12-25.

44. Sehgal R.R., Raj G. Interactive Voice Response Using Automatic Speech Recognition Techniques for Call Centers //Proceedings of 3rd International Conference on Internet of Things and Connected Technologies (ICloTCT). - 2018. - C. 26-27.

45. Stepanov E. et al. Automatic summarization of call-center conversations //Conference: IEEE Automatic Speech Recognition and Understanding Workshop (ASRU2015). - 2015.

46. Suciu G. et al. Social Media Cloud Contact Center Using Chatbots //International Conference on Future Access Enablers of Ubiquitous and Intelligent Infrastructures. - Springer, Cham, 2019.-C. 437-442.

47. Szymański P. et al. WER we are and WER we think we are //arXiv preprint arXiv:2010.03432. — 2020.

48. Tarasiev A. et al. Development of an intelligent automated system for dialogue and decision-making in real time //2018 2nd European Conference on Electrical Engineering and Computer Science (EECS). - IEEE, 2018. — C. 387-391.

49. The Gartner 2021 Predictions: Accelerate Results Beyond RPA to Hyperautomation // gartner.com: market research and business trend analytics URL: https:// www.gartner.com/en/webinars/3991832/the-gartner-2021-predictions-accelerate-results-beyond-rpa-to-hy (дата обращения: 28.01.2021).

50. Vasilateanu A., Ene R. Call-Center Virtual Assistant Using Natural Language Processing and Speech Recognition // Journal of ICT, Design, Engineering and Technological Science. 2018. Vol.2, No.2. C. 40-46.

51. Völkle C., Planing P. Digital Automation of Customer Contact Processes-an Empirical Research on Customer Acceptance of different Chatbot Use-cases // Digitalen Wandel gestalten. - Springer Gabler, Wiesbaden, 2019. - C. 217-229.

52. Wang L. et al. Voice-Based Al in Call Center Customer Service: Evidence from a Field Experiment //Call Center Customer Service: Evidence from a Field Experiment (June 22, 2020). - 2020.

53. Why Contact Centers Still Need People in the Digital Age // bcg.com: business analysis and decision-making URL: https://www.bcg.com/publications/2017/ operations-technology-contact-centers-people-digital-age (дата обращения: 28.01.2021).

54. «Тинькофф» интегрировал в телефонный колл-центр голосового помощника Олега // comnews.ru: информ. портал URL: https://www.comnews.ru/ digital-economy/content/212814/2021-01-28/2021-w04/tinkoff-integriroval-telefonnyy-koll-centr-golosovogo-pomoschnika-olega (дата обращения: 28.01.2021).

55. Будущее контактных центров. Перспективы развития индустрии до 2023 года // callcenterguru.ru: специализ. информ.-аналит. портал URL: https:// callcenterguru.ru/cc365/publications/554 (дата обращения: 28.01.2021).

56. Глобальное бенчмаркинговое исследование контактных центров. Россия и страны региона — 2019/2020. Автоматизация и роботизация: глава аналит. отчёта // contact-center-benchmark.ru: сайт иссл. рынка контакт-центров URL: https://contact-center-benchmark.ru/report-2020/chapter-5/ (дата обращения: 28.01.2021).

57. Демушкин А.А. Анализ существующих сервисов и средств для распознавания речи //Синтез междисциплинарного научного знания как фактор развития современной науки. - 2020.— - . 65-70.

58. Золотухина Е.Б., Голубева А.И. Автоматизация контактного центра с использованием инструментов омниканальности //Вектор экономики.2020.—№. 3.-C. 52-52.

59. Контакт-центр Al: основные сведения о программном решении // neuro.net: офиц. сайт URL: https://neuro.net/ru\#rec184351872 (дата обращения: 28.01.2021).

60. Неустроев М.Ю. Оценка эффективности работы центра обслуживания вызовов с использованием аналитики больших данных //Информационно-технологический вестник. - 2019.— № . 2.- С. 127-136.

61. Седойкина А.А. Перспективу внедрения системы искусственного интеллекта для повышения эффективности бизнес-процессов в саll-центре //Журнал «Human Progress» http://progress-human. com. — 2020.— T. 6. — № . 2 .

(c) Капусткин Максим Владимирович ( kapustkin.mv@edu.spbstu.ru ).

Журнал «Современная наука: актуальные проблемы теории и практики» 